\title{
Pantoprazole-induced acute interstitial nephritis
}

\author{
Sheila Klassen MD, Joan C. Krepinsky MD, Ally P.H. Prebtani MD
}

Competing interests: Ally Prebtani is a consultant for Novo Nordisk, EMD

Serono, Eli Lilly and

Sanofi; has received grants from Novo Nordisk, EMD Serono, Eli Lilly, Sanofi, Astra, Bristol-Myers Squibb and Pfizer; has received payment for lectures from Novo Nordisk, EMD Serono, Eli Lilly, Sanofi, Astra and Bristol-Myers Squibb; and has received payment for educational presentations from EMD Serono. No other competing interests were declared.

This article has been peer reviewed.

Correspondence to: sheila.liu.klassen@gmail .com

CMAJ 2013. DOI:10.1503 /cmaj.120954
$\Lambda$ 73-year-old man presented to the emergency department feeling tired and nauseous, and unable to eat. His medical history included congestive heart failure, stable rheumatoid arthritis and benign prostatic hypertrophy. He had not recently had any fever, rash or joint pain, nor did he have symptoms suggesting renal or systemic infections, prostatic obstruction or worsening congestive heart failure.

The patient's medications, which had been mostly unchanged for several months, included furosemide, ramipril and sulfasalazine. He had stopped taking tamsulosin 1 week before presenting to the emergency department, and he had started taking pantoprazole for gastroesophageal reflux disease 2 months earlier. He was not using any nonsteroidal anti-inflammatory drugs or antibiotic agents (including sulfonamides).

A physical examination showed a flat jugular venous pressure, dry mucous membranes and poor skin turgor, suggesting dehydration. The patient was afebrile and did not have costovertebral tenderness, a palpable bladder or skin rashes.

Results from initial laboratory investigations showed elevated creatinine (474 [normal 5090] $\mu \mathrm{mol} / \mathrm{L})$ and urea (29.5 [normal 2.5-8.0] $\mathrm{mmol} / \mathrm{L}$ ) levels. Results from blood tests done 1 month earlier showed levels closer to or within the normal ranges $(92 \mu \mathrm{mol} / \mathrm{L}$ creatinine, $7.3 \mathrm{mmol} / \mathrm{L}$ urea).

Renal ultrasonography done on admission to hospital was normal. An indwelling catheter was

\section{KEY POINTS}

- The number of cases of acute interstitial nephritis induced by proton pump inhibitors is increasing owing to the widespread use of these medications.

- Presenting symptoms are nonspecific, although urine leukocytes or eosinophils can be indicative; biopsy remains the gold standard for diagnosis.

- Early discontinuation of the culprit medication is crucial in preventing chronic renal failure.

- Steroid treatment is controversial and has inconsistent benefits, but the literature suggests more favourable outcomes with a tapering regimen.

inserted without much diuresis, and tamsulosin was restarted. The patient's furosemide and ramipril were withheld, and his sulfasalazine was switched to leflunomide to eliminate nephrotoxic medications. He was rehydrated with isotonic crystalloids over the next several days. The patient's creatinine level decreased to $251 \mu \mathrm{mol} / \mathrm{L}$ after 8 days.

Intravenous fluids were stopped on day 9 of the patient's stay in hospital because he was euvolemic and drinking well, and voiding well without the indwelling catheter. However, the patient's creatinine level slowly began to increase. The trend of his creatinine levels is shown in Figure 1. Urinalysis showed trace amounts of protein, $0-3$ red cells and more than 30 white cells (reference range $0-3$ ) per highpower field. A repeat urinalysis showed an elevated number of eosinophils. After consultation with a nephrologist, we considered a diagnosis of acute interstitial nephritis caused by pantoprazole; we stopped the medication on day 15 of the patient's stay in hospital.

A core biopsy specimen of the patient's right kidney showed diffuse edema of the interstitium with infiltration by lymphocytes, occasional eosinophils and plasma cells, as well as minimal interstitial fibrosis (Figure 2; Appendix 1, available at www.cmaj.ca/lookup/suppl/doi:10.1503 /cmaj.120954/-/DC1). The patient's interlobar arteries showed signs of nephrosclerosis. Immunofluorescence was negative for immunoglobulins A, G and M, and showed small granules of complement component 3 within vessel walls. Electron microscopy showed that the patient's glomeruli were normal. Overall, the findings were consistent with moderate acute interstitial nephritis.

The patient was discharged with a downwardtrending creatinine level, and he was provided with follow-up in the nephrology clinic. He did not receive prednisone. Forty days after he stopped taking pantoprazole, the patient's creatinine level was $133 \mu \mathrm{mol} / \mathrm{L}$, which decreased to $117 \mu \mathrm{mol} / \mathrm{L}$ on subsequent visits to the clinic.

Using the Naranjo probability score ${ }^{1}$ for adverse drug reactions, which takes into account 
factors such as temporal causality, known drug effects and alterative causes of the event, our patient's case was consistent with a "probable adverse drug reaction."

\section{Discussion}

Pantoprazole-induced acute interstitial nephritis is an increasingly known complication of proton pump inhibitor therapy. In our patient's case, confounding factors delayed diagnosis; on admission, volume depletion and possibly acute tubular necrosis contributed to his renal failure. Although rehydration and discontinuation of known nephrotoxic medications led to improved renal function, full resolution did not occur, and the patient's creatinine level began to rise when intravenous fluids were stopped.

Several factors were considered in our diagnosis, including the exclusion of other causes of acute renal failure, the presence of sterile pyuria with eosinophiluria on urinalysis, and the presence of acute interstitial nephritis on renal biopsy. The absence of any other pathology on biopsy, including acute tubular necrosis, renders other diagnoses very unlikely. Although our patient had been taking other medications, only pantoprazole is known to be associated with acute interstitial nephritis. Furthermore, the patient's creatinine level declined after he stopped taking pantoprazole.

\section{Background}

Acute interstitial nephritis is a glomerular-sparing form of nonoliguric kidney disease resulting from an allergic or immunologic reaction to intrarenal antigens and leading to tubular damage. The condition is commonly encountered as an adverse drug reaction, and most cases induced by medications are attributed to $\beta$-lactam antibiotic agents. Other common culprits include nonsteroidal antiinflammatory drugs, allopurinol, famotidine, furosemide and valproic acid. ${ }^{2}$

Acute interstitial nephritis induced by pantoprazole was first reported in 2004. ${ }^{3}$ From that report until January 2012, according to the World Health Organization's Uppsala Monitoring Centre (www.who-umc.org), there have been 87 cases of pantoprazole causing acute renal failure. In Canada, there have been 10 spontaneously reported cases of tubulointerstitial nephritis induced by proton pump inhibitors since $1965 .{ }^{4}$ In a retrospective case review, however, Geevasinga and colleagues found 90 cases of acute renal failure associated with proton pump inhibitors over a 14-year span at a single institution, ${ }^{5}$ suggesting that this complication may not be as infrequent as previously thought. Possible reasons for the discrepancy between the number of cases found in retrospective studies and those spontaneously reported are physicians not knowing that these drugs can cause acute interstitial nephritis and variability in the clinical presentation of the condition.

Although this adverse reaction has been observed as a class effect, there have been many more cases of acute interstitial nephritis related to the use of omeprazole. The reason for this is unclear and may simply be due to omeprazole's

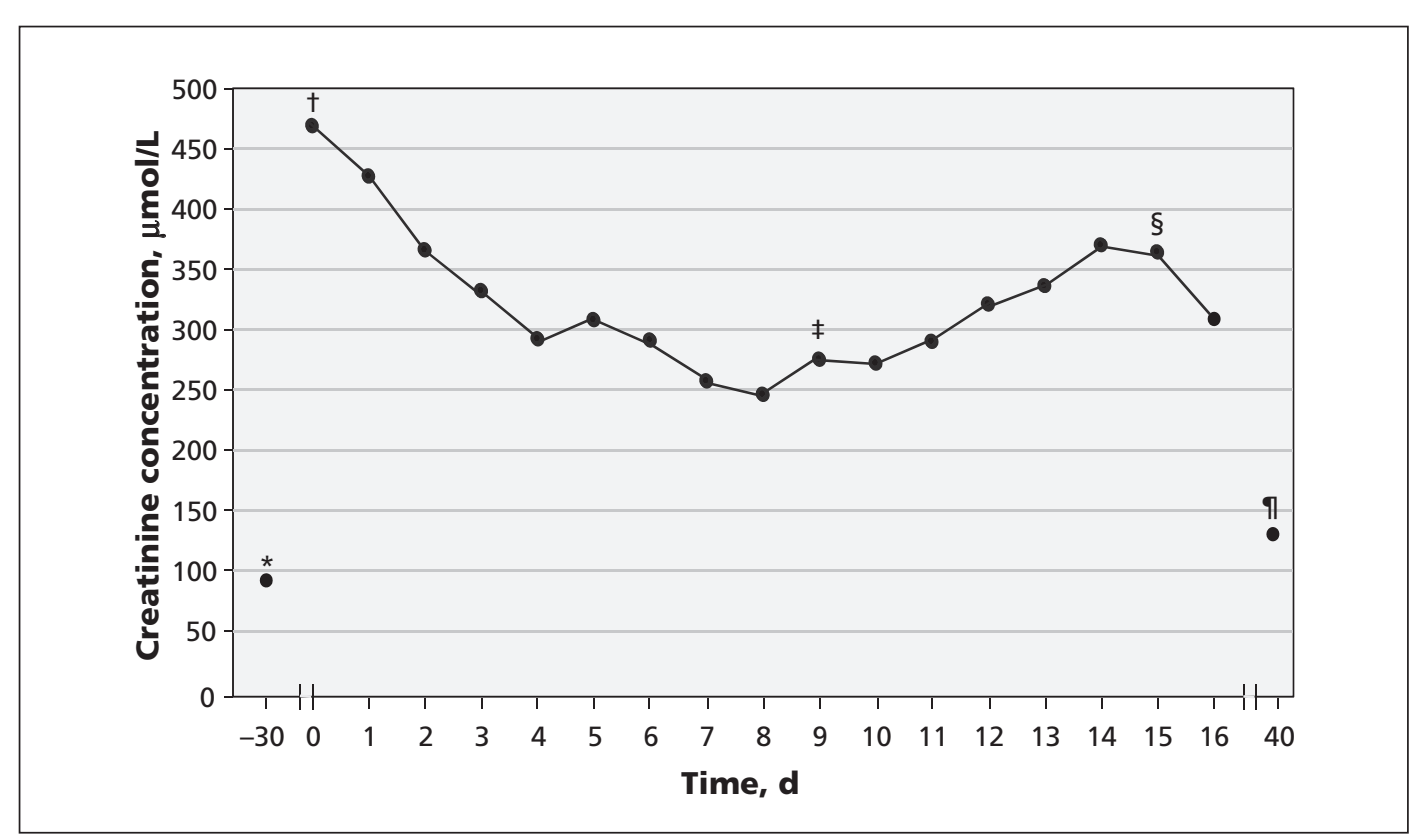

Figure 1: Patient's creatinine levels 1 month before and during admission. *One month before admission. tAdmission to hospital; furosemide, sulfasalazine and ramipril stopped, intravenous fluids started. ‡Intravenous fluids stopped. §Pantoprazole stopped. ๆResolution. 
longer availability on the market, lower specificity in its binding sites and lower molecular stability compared with other proton pump inhibitors. ${ }^{3}$

\section{Presentation and diagnosis}

The mean duration of exposure to proton pump inhibitors before the onset of acute renal failure is 3 months, although it has been reported to have occurred within hours. ${ }^{2,3}$ The mean age of presentation is 74 years, ${ }^{5}$ possibly because of the frequency with which these drugs are used by older patients.

Typical presenting symptoms of acute interstitial nephritis are nonspecific, such as fatigue and malaise. Acute renal failure is the only consistent clinical presentation, although oliguria is unusual. Nausea and vomiting are present in one-third of cases. ${ }^{5}$ The classic triad of fever, rash and eosinophilia, which may occur within several weeks of onset of the disease, is present in only $5 \%-33 \%$ of cases, making a clear diagnosis difficult. ${ }^{2}$

Although urea and creatinine levels are consistently elevated, no other laboratory or radiographic findings can confirm the diagnosis. Urinalysis is usually uninformative, with sterile pyuria being the most common abnormal finding. The level of proteinuria is very rarely in the nephrotic range. Eosinophils in the urine have been reported in as many as one-half of cases. More recently, microscopic hematuria has been reported in almost two-thirds of all cases of

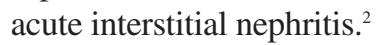

Gallium-67 imaging has been proposed to

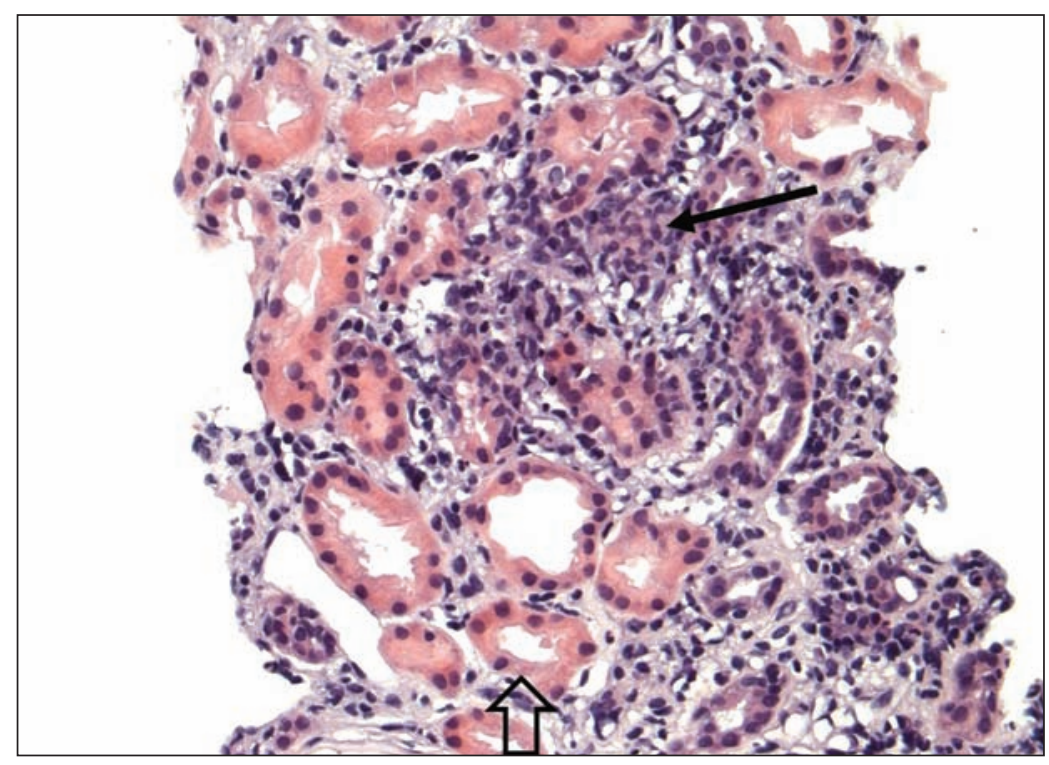

Figure 2: Core biopsy specimen of the patient's right kidney showing inflammatory infiltrate, predominantly lymphocytes, with interstitial edema separating the renal tubules (outlined arrow). There is a small granuloma formed in an area of dense interstitial inflammatory infiltrate (black arrow), suggesting an allergic cause. Hematoxylin and eosin stain (200× magnification). help differentiate between acute tubular necrosis and acute interstitial nephritis. Both disease processes may present similarly, with nonspecific symptoms. However, the distinction between the two is important. There is definitive management for acute interstitial nephritis, whereas the management of acute tubular necrosis is mainly supportive care. In a nuclear medicine study that reviewed 500 gallium-67 images, $5.6 \%$ of patients showed abnormal uptake at 48 hours. ${ }^{6}$ Of these patients, $18 \%$ had acute interstitial nephritis and $2 \%$ had acute tubular necrosis. However, glomerulonephritis, pyelonephritis, chronic interstitial nephritis and normal kidneys have shown false-positive results with this technique. ${ }^{6}$ The usefulness of gallium-67 imaging remains controversial; this technique may be useful in patients who have a good pretest likelihood of acute interstitial nephritis and who are too ill to tolerate renal biopsy.

Renal biopsy remains the gold standard for diagnosis. Typical histopathologic findings include interstitial edema with mononuclear cells, T lymphocytes, eosinophils and plasma cell infiltration around the renal tubules, sparing the glomeruli and blood vessels. Initially, interstitial fibrosis is mild and diffuse, but may progress to tubular atrophy or extensive interstitial fibrosis and glomerulosclerosis.

\section{Treatment and prognosis}

The most important aspect of treating acute interstitial nephritis is timely discontinuation of the offending agent. Removal of the trigger prevents further interstitial inflammation and tubular damage, with possible full recovery of renal function in 3-18 months. The role of steroids in treating acute interstitial nephritis is controversial. Although the results of several small trials suggest that patients given prednisone have better renal recovery, there have not been any large trials showing clear benefit. Methylprednisolone pulses given intravenously (250-500 mg/d for 3$4 \mathrm{~d}$ ), followed by a tapering course of prednisone (0.5-1 $\mathrm{mg} / \mathrm{kg}$ daily) over 4-6 weeks has been suggested..$^{2,7}$ The most recent retrospective multicentre study examining steroid use in druginduced acute interstitial nephritis confirmed by biopsy found that patients not given steroid therapy $(n=9)$ had significantly higher creatinine levels at the end of the follow-up period than patients who had received treatment $(n=52) .^{7}$ In addition, patients who stopped taking the offending drug and began steroid treatment more than 7 days after the onset of renal failure had a 6fold increased risk of chronic renal disease. ${ }^{7} \mathrm{~A}$ similar retrospective study involving 60 patients did not show improved recovery of renal func- 
tion with corticosteroids, although there was a delay of 3-4 weeks after the onset of renal failure before steroid treatment began. ${ }^{8}$ A substantial proportion of patients in both groups had elevated creatinine levels at follow-up. ${ }^{8}$ Interestingly, a case study noted that despite continuing pantoprazole in a patient with acute interstitial nephritis, a 2-month course of high-dose prednisone therapy with tapering eradicated inflammatory infiltrates on renal biopsy. ${ }^{9}$ The authors also noted that the histologic appearance did not change on subsequent biopsy at 3 months after pantoprazole had been withdrawn and prednisone continued at $20 \mathrm{mg} / \mathrm{d} .^{9}$

There has been no clear correlation between the length of exposure to proton pump inhibitors and duration or severity of acute renal failure after resolution of acute interstitial nephritis. In a small proportion of cases, if extensive tubular damage had occurred, permanent dialysis was required.

\section{Conclusion}

Because the link between proton pump inhibitors and interstitial nephritis is just beginning to emerge, a high index of suspicion is needed to diagnose drug-induced acute interstitial nephritis. As previously suggested, the infrequency of the diagnosis may be due to a lack of awareness of this adverse effect rather than to the infrequency of allergic nephritis itself. Because many patients presenting with this condition are already in hospital for infections and illness and taking medications that are more commonly known to cause renal failure, the diagnosis of acute interstitial nephritis cannot be made unless physicians are aware that it is an adverse effect associated with proton pump inhibitors.

Prospective randomized trials comparing simple withdrawal of offending medication with withdrawal of medication plus steroid therapy, in addition to trials comparing low- and high-dose steroid therapy, would be valuable in guiding the treatment of this condition.

\section{References}

1. Naranjo CA, Busto U, Sellers EM, et al. A method for estimating the probability of adverse drug reactions. Clin Pharmacol Ther 1981;30:239-45

2. Praga M, Gonzalez E. Acute interstitial nephritis. Kidney Int 2010; 77:956-61.

3. $\mathrm{Ra} \mathrm{A}$, Tobe SW. Acute interstitial nephritis due to pantoprazole. Ann Pharmacother 2004;38:41-5.

4. MedEffect Canada. Canada Vigilance Adverse Reaction Online Database. Ottawa (ON): Health Canada; 2012. Available: www .hc-sc.gc.ca/dhp-mps/medeff/databasdon/index-eng.php (accessed 2012 Aug. 3)

5. Geevasinga N, Coleman PL, Webster AC, et al. Proton Pump Inhibitors and Acute Interstitial Nephritis. Clin Gastroenterol Hepatol 2006;4:597-604.

6. Lin DS, Sanders JA, Patel BR. Delayed renal localization of Ga67. J Nucl Med 1983;24:894-7.

7. González E, Gutierrez E, Galeano C, et al. Early steroid treatment improves the recovery of renal function in patients with druginduced acute interstitial nephritis. Kidney Int 2008;73:940-6.

8. Clarkson MR, Giblin L, O'Connell FP, et al. Acute interstitial nephritis: clinical features and response to corticosteroid therapy. Nephrol Dial Transplant 2004;19:2778-83.

9. Kim MJ, Heim M, Mayr M. Effect of corticosteroids during ongoing drug exposure in pantoprazole-induced interstitial nephritis. Nephrol Dial Transplant 2010;25:1716-9.

Affiliation: From the Department of Medicine (Klassen, Krepinsky, Prebtani), McMaster University, Hamilton, Ont.

Contributors: Sheila Klassen was the primary author of the paper. Joan Krepinsky and Ally Prebtani reviewed the paper and revised it for important intellectual content. All of the authors approved the final version of the manuscript submitted for publication.

Acknowledgements: The authors would like to thank Dr. Iakovina Alexopoulou, Department of Pathology, McMaster University, for her assistance in the pathology of this case.

The Canadian Vigilance Program is a useful resource containing spontaneously reported post-marketing adverse drug reactions, and is a way in which physicians can contribute to drug safety. It can be found at www.hc-sc.gc.ca/dhp -mps/medeff/databasdon/index-eng.php.

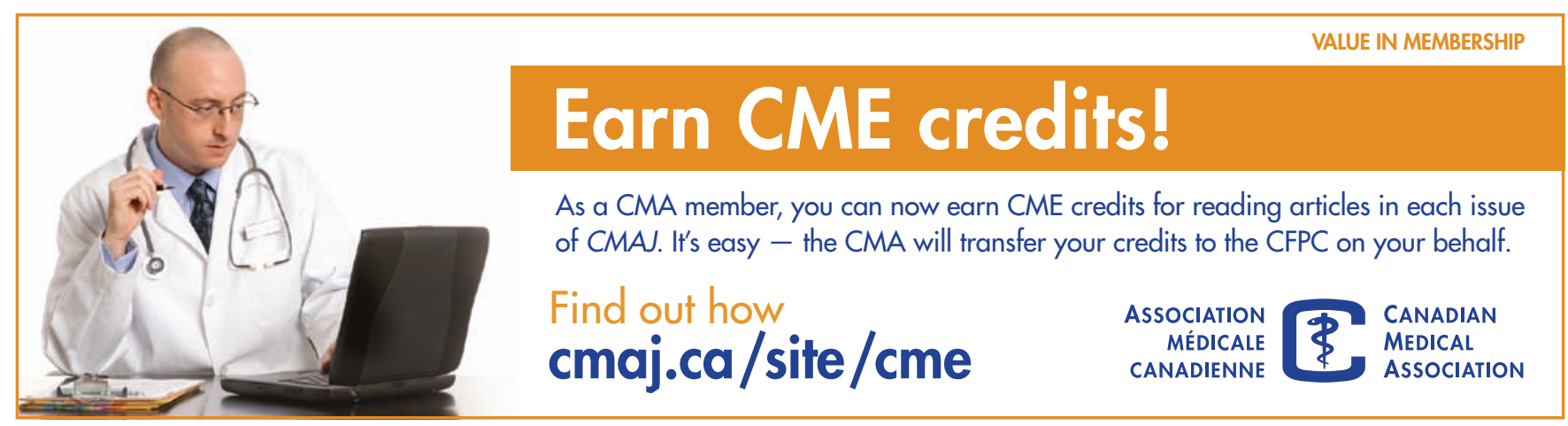

